\title{
Polymerization of 2-Methacryloyloxyethyltrimethylammonium Chloride Organized on Poly(sodium acrylate) Template
}

\author{
Shuzo AOKI and Yoshihiro KiHaRa \\ Department of Applied Chemistry, Faculty of Engineering, Osaka City University, \\ Sumiyoshi-ku, Osaka 558, Japan
}

(Received April 4, 1996)

\begin{abstract}
Methacryloyloxyethyltrimethylammonium chloride (MMAC) was polymerized in the presence of poly(sodium acrylate) as the template polymer and potassium persulfate as the initiator in water. The polymerization was accompanied by the precipitation of complex of poly(MMAC) and poly(sodium acrylate). A negative template effect (polymerization rate reduction) was observed when the template of low degree of polymerization $\left(P_{n}\right)$ was used, while increase of $P_{n}$ of the template decreased such negative effect. Coexistence of the template and sodium chloride to increase ionic strength of the reaction medium showed great negative template effect even by using the template of high $P_{n}$. The results were interpreted based on zip and pick-up mechanisms. The polymerization of MMAC took place thermally in the presence of the template of high $P_{n}$, indicating the effect of organization of the monomer.

KEY WORDS Radical Polymerization / Quaternary Ammonium Monomer / Polyanion Template / Organized Reaction System /
\end{abstract}

The polymerization behavior of vinyl monomers in organized states, such as micelles, vesicles, liquid crystals, etc., has been predicted to be greatly different from that in a molten state (bulk) or isotropic solution, and many polymer chemists are strongly interested in studying such organized polymerization reactions. ${ }^{1}$

Template polymerization, in which charged monomer molecules are regularly arranged on template polymer chains having opposite charge by Coulombic interaction, is a typical strictly organized reaction. ${ }^{2,3}$ In a template polymerization system, the monomer becomes highly concentrated state at the reaction site regardless of its concentration in the recipe, and the appropriately organized state facilitates the polymerization as a positive template effect (polymerization rate enhancement).

4-Vinylpyridine spontaneously polymerizes to give the polymer consisting of an ionen-type structure in the presence of poly(acrylic acid) as the template in water, ${ }^{4,5}$ while in the presence of poly(methacrylic acid) 2-vinylpyridine yields a polymer with ordinary structure. ${ }^{6} \mathrm{Al}-$ though poly(potassium acrylate) is not able to initiate the polymerization of 4-vinylpyridine, it favors the propagation in polymerization under $\gamma$-irradiation. ${ }^{7}$ The template polymerization of vinylpyridines gives complicated results especially due to the initiation mechanism containing both ionic and radical intermediates characteristic of the aqueous polymerization of 2- and 4-vinylpyridines. ${ }^{8}$ To advantageously avoid problems due to such complicated initiation mechanism, it is required to use a monomer structurally impossible to be polymerized through a manner resulting in the ionen polymer.

This paper describes the polymerization behavior of 2-methacryloyloxyethyltrimethylammonium chloride (MMAC), which is polymerizable only via opening of the vinyl double bond, in combination with poly(sodium acrylate) as a template polyanion. Micelle formation of amphiphilic compounds in water is based on a hydrophobic interaction between long-chain alkyls of the amphiphile, while, in the present case, monomer-tem- plate complexation takes place by the electrostatic interactions between oppositely charged ionic groups. The present polymerization system would thus behave like an organized molecular aggregate, although MMAC is not amphiphilic or micelle-forming.

Recently we reported that the polymerization behavior of (meth)acrylate anions as counter ions of conventional cationic surfactant micelles in the place of template polymer chains ${ }^{9}$ was considerably different from that of their structural counterpart alkyl 2-methacryloyloxyethyldimethylammonium bromides with longchain alkyls (polymerizable groups exist inside micelles). ${ }^{10}$ Therefore, it is of interest to investigate further the effects of the mode of molecular aggregation and extent of restriction of polymerizable double bonds in the aggregate on the polymerization behavior.

\section{EXPERIMENTAL}

\section{Materials}

2-Methacryloyloxyethyltrimethylammonium chloride was used as provided by Mitsubishi Rayon Co. in high pure crystalline state. Poly(sodium acrylate) of low degree of polymerization offered by KAO Corp. was adjasted to its stoichiometric structure by adding sodium hydroxide solution and reprecipitated three times by water/methanol system. Poly(sodium acrylate)s of higher degree of polymerization were prepared by the radical polymerization of acrylic acid in hexane in the presence or absence of isopropanol, followed by stoichiometric neutralization with sodium hydroxide and purification in the same way above. The molecular weight of these acrylate polymers for use as templates was determined, after converting them to the methyl ester using diazomethane, by Tosoh 8000 series gel permiation chromatography using polystyrene standard. The results are listed in Table I.

Potassium persulfate (KPS) as the polymerization initiator was recrystallized from water, and water as the polymerization solvent was distilled after ion- 
Table I. Poly(sodium acrylate) template

\begin{tabular}{crrrr}
\hline Abb. of sample & \multicolumn{1}{c}{$M_{n}{ }^{\mathrm{a}}$} & \multicolumn{1}{c}{$M_{w}{ }^{\mathrm{b}}$} & $M_{w} / M_{n}$ & $P_{n}{ }^{\mathrm{c}}$ \\
\hline PSA 50 & 4410 & 7140 & 1.62 & 51 \\
PSA 200 & 16570 & 36470 & 2.20 & 193 \\
PSA 1500 & 131440 & 416300 & 3.17 & 1500 \\
\hline
\end{tabular}

${ }^{\text {a }}$ Number average molecular weight determined for methyl ester.

${ }^{b}$ Weight average molecular weight determined for methyl ester.

${ }^{c}$ Number average degree of polymerization.

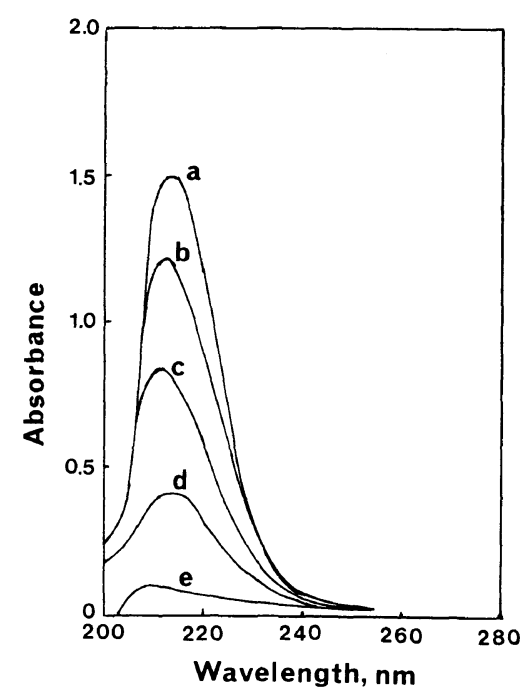

Figure 1. UV absorption spectra of a mixture of monomer, template polymer and preformed daughter polymer in $5.0 \mathrm{M} \mathrm{NaCl}$ aqueous solution at $[$ monomer $]+[$ daughter polymer $]=[$ template polymer $]=$ $2.0 \times 10^{-4} \mathrm{moll}^{-1}$. [monomer] $/[$ daughter polymer $]=1 / 0$ (a) $; 0.75 /$ 0.25 (b); $0.5 / 0.5$ (c); $0.25 / 0.75$ (d); 0/1 (e).

exchanged.

\section{Polymerization}

Polymerization was carried out in a sealed glass tube, after removing dissolved oxygen by the freeze-thaw technique, with shaking in the dark at a given temperature. The produced polymer (daughter polymer) precipitated by forming an insoluble stoichiometric interpolymer complex with the template polyanion. ${ }^{11}$ Monomer-to-polymer conversion was determined by decrease of UV absorption based on vinyl double bond $\left(\lambda_{\max }=212.8 \mathrm{~nm}\right)$ in the polymerization mixture, after the precipitated polymer complex was dissolved to obtain a clear solution by the addition of sodium chloride to increase ionic strength of the solution. The UV absorption spectra were recorded using a Shimadzu UV-160 spectrophotometer. Figure 1 shows the spectra of a mixture of preformed poly(MMAC) $\left([\eta]=0.564 \mathrm{dl} \mathrm{g}^{-1}\right.$ in $3 \mathrm{M} \mathrm{NaCl}$ at $30^{\circ} \mathrm{C}$ ) and template polymer (PSA50) at different ratios, and the calibration curve obtained from this figure for determining the conversion is shown in Figure 2. For other templates, almost the same results were obtained. The concentrations of polymers will be expressed based on molar concentrations of the repeating monomer units.

Attempt to separate the template and daughter polymers from the complex being insoluble in water and polar organic solvents, to characterize the daughter polymer, was unsuccessful. Although, by concentrated hydrochloric acid, it was possible to elute the cationic

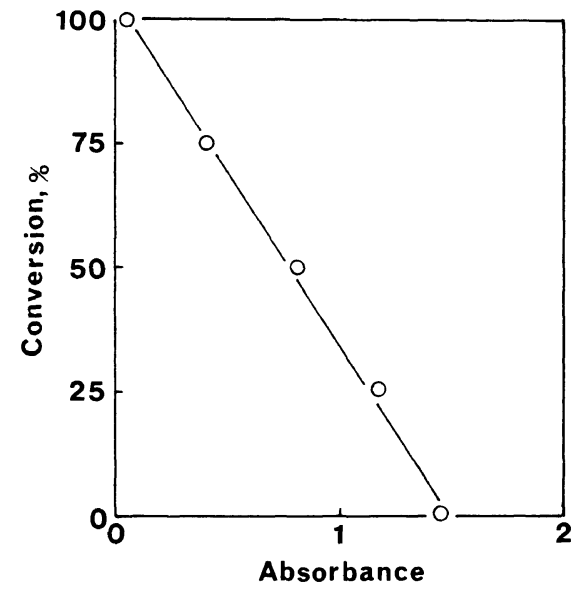

Figure 2. Relationship between UV absorbance at $212.8 \mathrm{~nm}$ and monomer-to-polymer conversion.

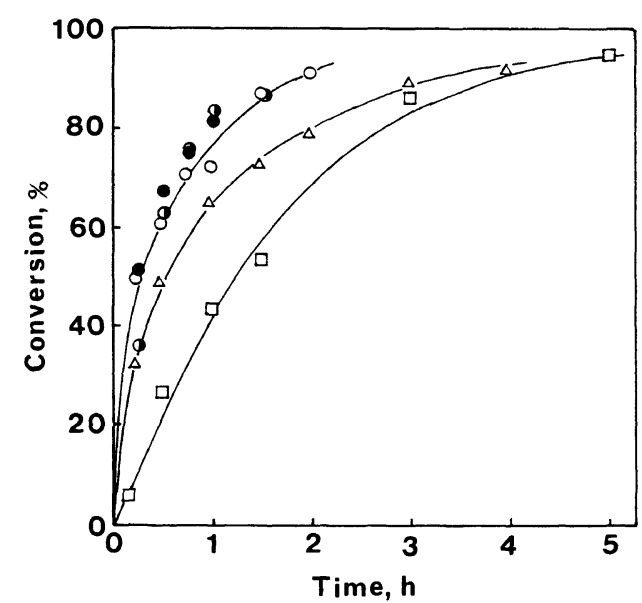

Figure 3. Time-conversion curves in the presence of template polymer with different $P_{n}$ at [monomer] $=$ [template polymer] $=0.1 \mathrm{moll}^{-1}$, $[\mathrm{KPS}]=0.005 \mathrm{moll}^{-1}$, and $60^{\circ} \mathrm{C}$. , absence of template; $\mathbf{C}$, sodium acetate; $\bigcirc, \operatorname{PSA} 1500 ; \triangle$, PSA $200 ; \square, \operatorname{PSA} 50$.

polymer separately, leaving the polyanion as an insoluble solid, from the complex prepared by admixing the preformed poly(MMAC) and template polymer solutions, the template polymerization products, especially those obtained in the presence of the template with lower $P_{n}$, could not be separated, because of the formation of inseparable opaque solution.

\section{RESULTS AND DISCUSSION}

\section{Effect of $P_{n}$ of Template Polymer}

The peroxide-initiated polymerization of MMAC was carried out in the absence or presence of the template at $60^{\circ} \mathrm{C}$ in water. Poly(sodium acrylate)s with different $P_{n}$, PSA50, PSA200, and PSA1500, were used as templates, and sodium acetate as the control $\left(P_{n}=1\right)$.

Time-conversion curves of the MMAC polymerization performed at a constant ratio, $\left[-\mathrm{COO}^{-}\right] /\left[-\mathrm{N}^{+}\right.$ $\left.\left(\mathrm{CH}_{3}\right)_{3}\right]=1 / 1$, are shown in Figure 3 . Since the presence of sodium acetate little affected the polymerization rate, the results of polymerization in the absence of added salt or polymer served as reference. The presence of the template with lower $P_{n}$ greatly suppressed the polymerization rate, while the template with higher $P_{n}$ did not. This means a negative template effect (rate reduction 
effect) inversely depending on $P_{n}$ in contrast to the generally accepted results (rate enhancement effect) in other systems. ${ }^{2,3}$ This negative effect is recognized as a polymer effect from the fact that the presence of sodium acetate does not affect the polymerization rate.

Matuszewska-Czerwik and Polowinski reported that, in the polymerization of methacrylic acid in the presence of poly(vinylpyrrolidone) template in water, the rate of polymerization was not affected by the template with short chain length $\left(P_{n}=c a .50\right)$, but increased with chain length of the template. ${ }^{12}$ This coincides apparently with that of the present case, at least, regarding rate enhancement.

\section{Effects of Ratio of Template and Monomer}

When PSA50 was used, the increase of $\left[-\mathrm{COO}^{-}\right] /$ $\left[-\mathrm{N}^{+}\left(\mathrm{CH}_{3}\right)_{3}\right]$ ratio from $1 / 1$ to $2 / 1$ showed no difference in the time-conversion curve (Figure $4 \mathrm{a}$ ), while decrease of $\left[-\mathrm{COO}^{-}\right]$to the ratios $0.75 / 1-0.25 / 1$ brought about suppression of the polymerization corresponding to the case of $1 / 1$ until about $75-25 \%$ conversion depending on amount of template. After that the polymerization rate restored to the case of no template (Figures $4 b-d$ ).

We assumed, as a basic concept in the present polymerization system, that the quaternary ammonium chloride monomer produces $1 / 1$ salt with poly(sodium acrylate) by double decomposition in water and then polymerizes according to the so-called zip mechanism, because of strong ionic interaction between the template
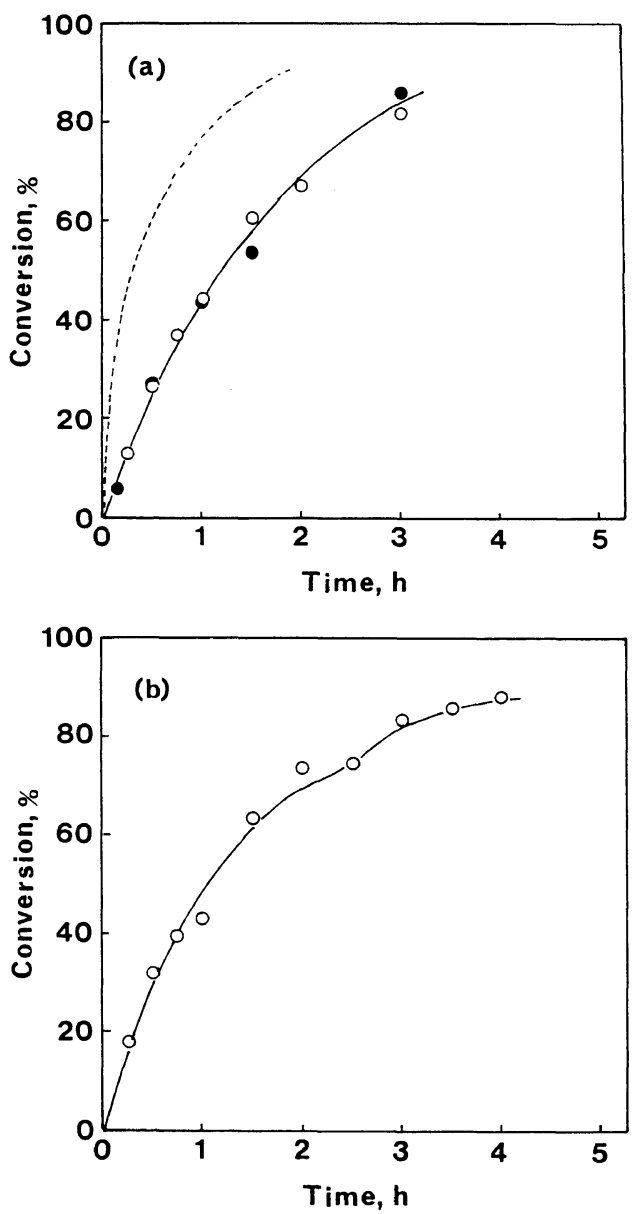

and the monomer as expected, favoring this mechanism. ${ }^{2,3}$ However this concept is contradicted from the results obtained at $\left[-\mathrm{COO}^{-}\right] /\left[-\mathrm{N}^{+}\left(\mathrm{CH}_{3}\right)_{3}\right]>1$ and $\left[-\mathrm{COO}^{-}\right] /\left[-\mathrm{N}^{+}\left(\mathrm{CH}_{3}\right)_{3}\right]<1$.

At first, in the case $\left[-\mathrm{COO}^{-}\right] /\left[-\mathrm{N}^{+}\left(\mathrm{CH}_{3}\right)_{3}\right]>1$, two distribution modes of the monomer molecules on the template chains may be possible; (a) the monomers bind randomly at anionic sites of all template chains to decrease the monomer concentration at the reaction site, and (b) the monomer binds cooperatively at anionic sites of the template to give the mixture of fully complexed and uncomplexed template chains as known in cationic surfactant-polyelectrolyte systems. ${ }^{13}$ In mode (a), to interpret the results in Figure 4a, the monomer molecules bound on the template must be able to move easily to unoccupied sites of the template with the propagation of polymerization. ${ }^{2,3}$ Although this requires large $k_{1}$ and $k_{2}$ in eq 1 for complex formation, we do not have any evidence. In mode (b), the cooperative process is questionable because this monomer does not have a hydrophobic moiety that would determine the binding site. ${ }^{13}$

Secondly, in the case $\left[-\mathrm{COO}^{-}\right] /\left[-\mathrm{N}^{+}\left(\mathrm{CH}_{3}\right)_{3}\right]<1$, complexed and free monomers exist. Since the free monomer has higher polymerizability than the complexed one from the results mentioned above (Figure 3 ), the free monomer must polymerize first at a faster rate at the initial stage and then the complexed monomer will polymerize in a slower rate after consumption of the free
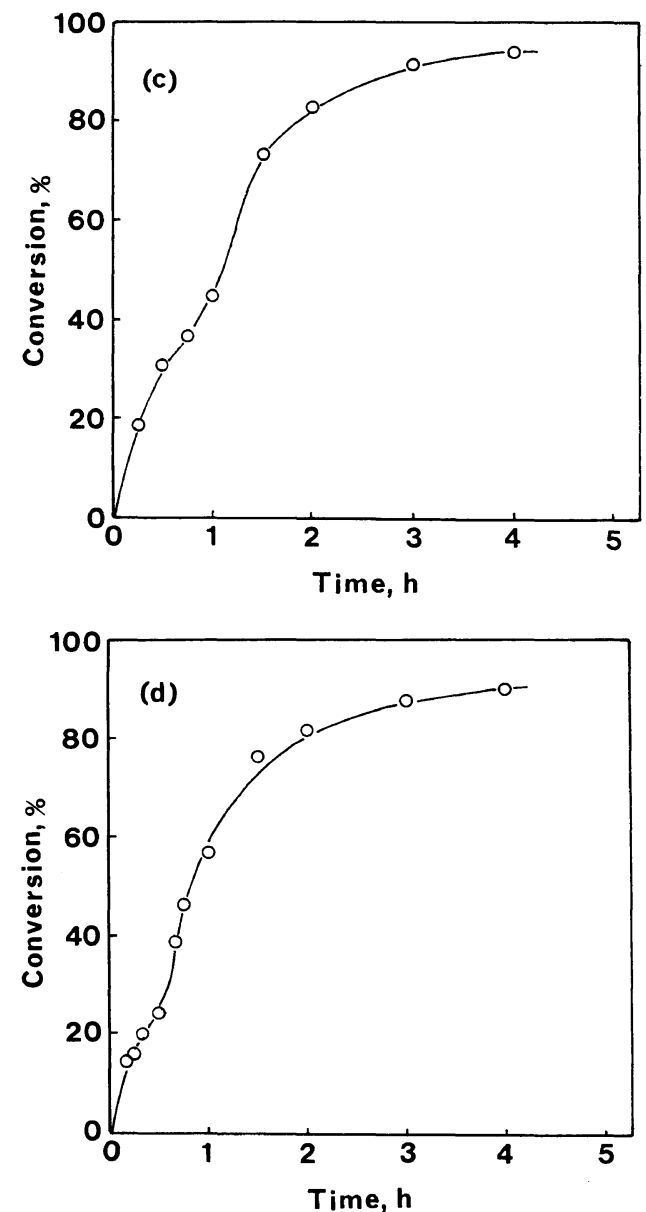

Figure 4. Time-conversion curves in the presence of template PSA50 at different mole ratios with the monomer $\left([\mathrm{monomer}]=0.1 \mathrm{~mol} 1^{-1}\right.$, $[\mathrm{KPS}]=0.005 \mathrm{moll}^{-1}$, and $\left.60^{\circ} \mathrm{C}\right)$.

$[\mathrm{PSA} 50] /[$ monomer $]=\mathrm{a}, \cdots 0, \bigcirc 2$, and $\bigcirc \mathrm{b}, 0.75 ; \mathrm{c}, 0.5 ; \mathrm{d}, 0.25$. 
monomer. The experimental results obtained (Figures $4 b-d$ ) were entirely the reverse (slower rate at the initial stage and faster rate at the later stage), indicating the simple zip mechanism is not acceptable for the present system.

The following discussion explains the experimental results. In eq 1 and 2 for complex formation between the template (-p-p-p-) and the monomer (M) or daughter polymer (-m-m-m-), equilibrium constant $K_{\mathrm{m}}$ would be larger than $K_{\mathrm{M}}$, because of a cooperative interaction between the polymer chains. ${ }^{2,3}$

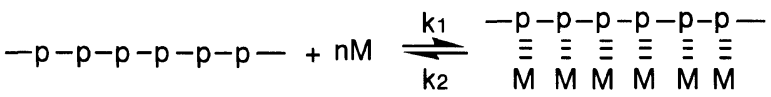

$$
\begin{aligned}
& \left(k_{1} / k_{2}=K_{\mathrm{M}}\right)
\end{aligned}
$$

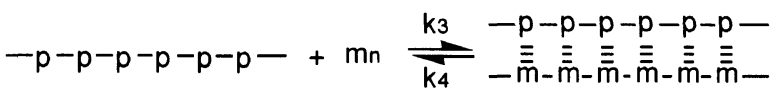

$$
\begin{aligned}
& \left(k_{3} / k_{4}=K_{\mathrm{m}}\right)
\end{aligned}
$$

With excess amounts of the monomer, the following reactions are possible.

Radical $(\mathrm{R} \cdot)$ formation by decomposition of the initiator (I)

$$
1 \rightarrow 2 R \text {. }
$$

Free monomer initiation

$$
R \cdot+M \rightarrow m \text {. }
$$

Complexed monomer initiation

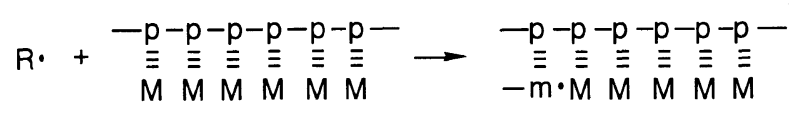

Free propagation

$$
m \cdot+n-1 m \rightarrow m n \cdot
$$

Complex exchange

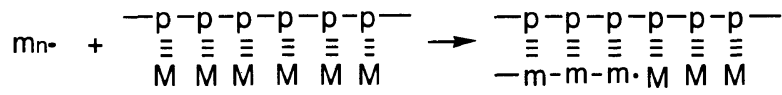

$$
\begin{aligned}
& +\mathrm{nM}
\end{aligned}
$$

Template propagation by zip mechanism

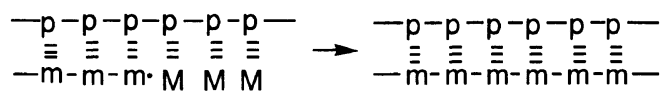

The addition of radical species $(R \cdot)$ produced by thermal decomposition of the initiator (I) takes place toward free or complexed monomer molecules (eq 3 and 4), followed by propagation (eq 5 and 7). The oligomer radical $\left(\mathrm{m}_{\mathrm{n}} \cdot\right)$, free from the template, which has attained to critical chain length, ${ }^{2,3}$ exchanges with monomer molecules of the complex according to eq 6 due to difference between equilibrium constants $K_{\mathrm{m}}$ and $K_{\mathrm{M}}$. The template propagation by zip mechanism (eq 7) proceeds exclusively with slower rate, until all template polymer chains form complexes with the daughter polymer chains (initial stage of the polymerization). After that, the polymerization system becomes free from the rate control by the template (later stage of the polymerization).

The effect of degree of polymerization mentioned above will be discussed again based on the mechanism mentioned above. If the template polymerization proceeds via zip mechanism, it becomes impossible to continue further propagation and this leads to mono-radical termination, when the growing radical arrives at the end of template chain. Thus, the rate of termination increases by decreasing the chain length of the template due to increasing frequency of the mono-radical termination. That this polymerization system becomes heterogeneous with progress of the reaction due to the formation of template-daughter polymer complex being insoluble in the reaction medium may also responsible for the mono-radical termination process.

Matuszewska-Czerwik and Polowinski conclude that rate enhancement by the template is due to greater decrease of the termination rate constant compared to that of the propagation rate constant. ${ }^{14,15}$ In the present system, it may be concluded that increase of ratio of termination to propagation rates causes the reduction of rate of polymerization.

Effects of Coexistence of Template and Sodium Chloride

The equilibrium constants $K_{\mathrm{M}}$ and $K_{\mathrm{m}}$ become smaller with increasing ionic strength of the solution by the addition of an inorganic electrolyte. This gives a drastic effect on the template polymerization.

The rate reduction effect with the template polymer vanished by the addition of sodium chloride above $[\mathrm{NaCl}] /\left[-\mathrm{N}^{+}\left(\mathrm{CH}_{3}\right)_{3}\right]=6$ in the polymerization system at $\left[-\mathrm{COO}^{-}\right] /\left[-\mathrm{N}^{+}\left(\mathrm{CH}_{3}\right)_{3}\right]=1 / 1$ (Figure 5), and the polymerization proceeded homogeneously because of the great increase of ionic strength of the reaction medium to prevent complex formation. At $[\mathrm{NaCl}] /$ $\left[-\mathrm{N}^{+}\left(\mathrm{CH}_{3}\right)_{3}\right]=2$, the templates, especially those having longer chain length, strongly suppressed the polymerization. In the absence of the template, the change of the polymerization rate by the addition of sodium chloride was not so notable, while the solution viscosity, $[\eta]_{3 \mathrm{MNaCl}}^{30^{\circ} \mathrm{C}}$, of poly(MMAC) was reduced from 0.564 ([NaCl]/ $[$ monomer $]=0)$ to $0.364 \mathrm{dl} \mathrm{g}^{-1}([\mathrm{NaCl}] /[$ monomer $]=$ 2).

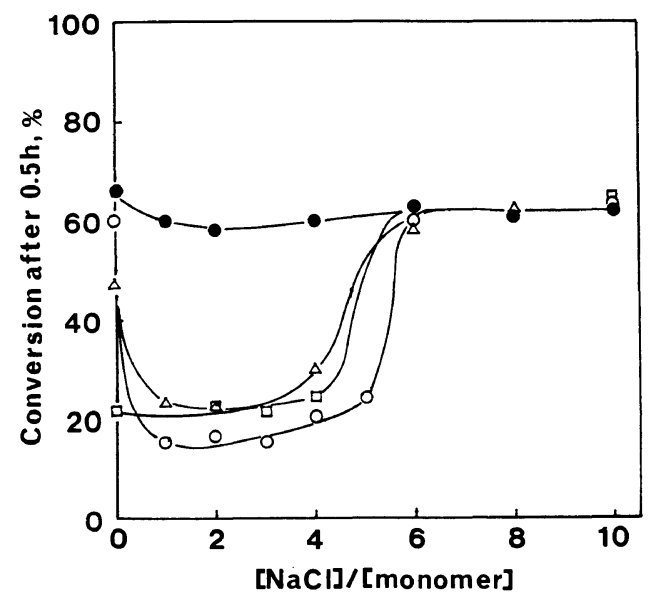

Figure 5. Effects of sodium chloride at $[$ monomer $]=[$ template polymer $]=0.1 \mathrm{moll}^{-1},[\mathrm{KPS}]=0.005 \mathrm{moll}^{-1}$, and $60^{\circ} \mathrm{C}$. absence of template; $\bigcirc$, PSA $1500 ; \triangle$, PSA200; $\square$, PSA50. 


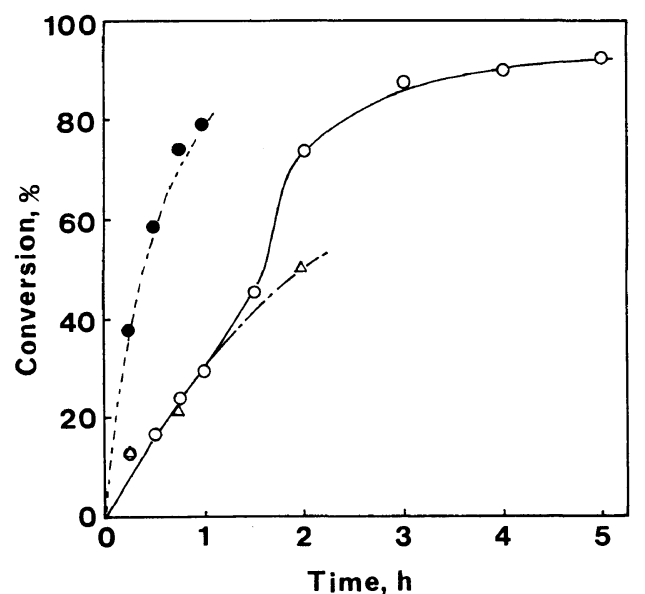

Figure 6. Time-conversion curves in the presence of template PSA 1500 at different mole ratios with the monomer in aqueous sodium chloride solution at $[\mathrm{monomer}]=0.1 \mathrm{moll}^{-1},[\mathrm{KPS}]=0.005 \mathrm{moll}^{-1}$, $[\mathrm{NaCl}]=0.2 \mathrm{moll}^{-1}$, and $60^{\circ} \mathrm{C}$. [PSAl 500$] /[\mathrm{monomer}]=0 \quad \mathrm{O} ; 1 \triangle$; $0.5 \bigcirc$.

Figure 6 shows the time-conversion curves of the polymerization systems consisting of monomer-sodium chloride-PSA1500 ( $1: 1: 0,0.5$, and 1 in molar ratio) in water. The ratio $\left[-\mathrm{COO}^{-}\right] /\left[-\mathrm{N}^{+}\left(\mathrm{CH}_{3}\right)_{3}\right]=0.5 / 1$ caused rate reduction until all template chains formed complexes with the daughter polymer chains, and then the rate of polymerization restored to the case of no template, as observed in Figures $4 \mathrm{~b}-\mathrm{d}$. This indicates that the above relation between the rate constants of propagation and termination holdes even in the presence of sodium chloride.

In the polymerization behavior observed in the presence of sodium chloride at the ratios $[\mathrm{NaCl}] /\left[-\mathrm{N}^{+}\right.$ $\left.\left(\mathrm{CH}_{3}\right)_{3}\right]=1-4$, the effect of template chain length disappeared. The polymerization system became heterogeous with progress of the reaction, showing complex formation between the template and daughter polymer to still occur in this sodium chloride concentration range, although $K_{\mathrm{M}}$ and $K_{\mathrm{m}}$ would become smaller. The zip mechanism applied in the absence of sodium chloride is not acceptable for the present system of high ionic strength. The probability of free monomer initiation in eq 3 increases with ionic strength. The growing oligomer radical which attained critical chain length, being longer than that in lower ionic strength reaction medium, forms a complex according to the equilibrium constant $K_{\mathrm{m}}$, where the pick-up mechanism ${ }^{2,3}$ (eq 8) seems to be applicable. Subsequently template propagation proceeds.

Template propagation by pick-up mechanism

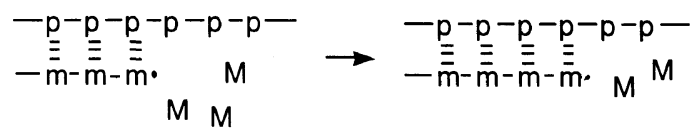

The propagation rate by eq 8 seems slower than that by eq 7 , because of the difference in local monomer concentration near the growing polymer radial on the template. The growing polymer radical on the template reacts with another growing polymer radical in bulk to terminate the polymerization (eq 9) in competition with the propagation. Thus the negative template effect is stronger.

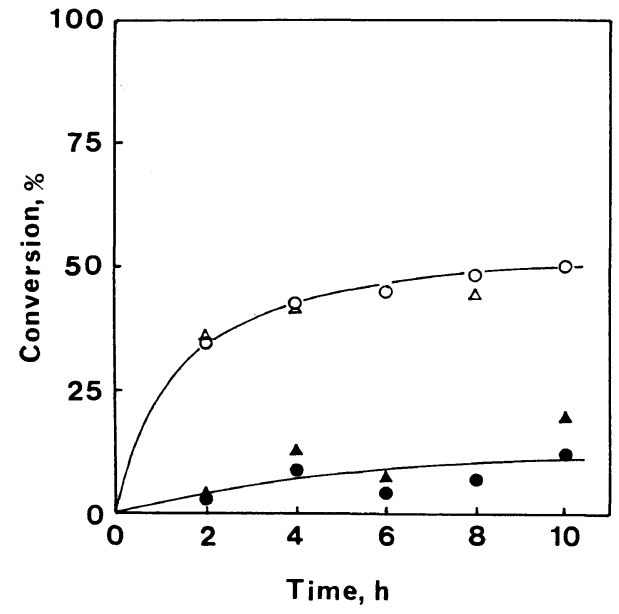

Figure 7. Thermal polymerization in the presence of template polymer at $[$ monomer $]=[$ template polymer $]=0.1 \mathrm{moll}^{-1}$. $O$, PSA 1500 at $60^{\circ} \mathrm{C} ; \triangle$, PSA 1500 at $80^{\circ} \mathrm{C} ; \boldsymbol{O}$, PSA 50 at $60^{\circ} \mathrm{C} ; \Delta$, PSA 50 at $80^{\circ} \mathrm{C}$.

Bi-radical termination

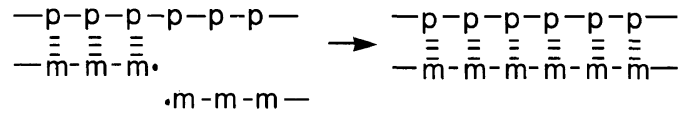

\section{Spontaneous Polymerization}

We reported that some amphiphilic monomers polymerized spontaneously with the formation of organized aggregates, such as micelles and liquid crystals. The initiation of polymerization should occur with a radical species produced by thermal reaction of the monomer molecules. The extent of restriction of the monomer molecules in the aggregates is closely related to the ease of radical formation. ${ }^{16,17}$

Micelle-forming alkyl 2-methacryloyloxyethyldimethylammonium bromides with a long-chain alkyl (the polymerizable double bonds exist inside micelles) easily polymerize spontaneously above the cmc in water. ${ }^{10}$ Alkyltrimethylammonium methacrylate, a polymerizable double bond outside micelles, is not able to polymerize spontaneously. ${ }^{9}$ In the present case, 2-methacryloyloxyethyltrimethylammonium ions were arranged on the template in a kind of aggregation.

Polymerization was carried out in the presence of the template at $[$ monomer $]=0.1 \mathrm{moll}^{-1},\left[-\mathrm{COO}^{-}\right] /\left[-\mathrm{N}^{+}\right.$ $\left.\left(\mathrm{CH}_{3}\right)_{3}\right]=1 / 1$, and 60 or $80^{\circ} \mathrm{C}$, and proceeded at an appreciable rate, as shown in Figure $7 .{ }^{18}$ The template PSA 1500 was particularly effective, while in the absence of the template no polymerization was observed even after $10 \mathrm{~h}$ at $80^{\circ} \mathrm{C}$. The difference in activity inducing MMAC polymerization was observed between templates with high and low $P_{n}$, similar to Figure 3 , presumably for the same reason of peroxide-initiated polymerization. There was no difference in the time-conversion curves with polymerization temperature 60 or $80^{\circ} \mathrm{C}$, and conversion was limited at a rather early stage of polymerization. The cause of these characteristics is still not elucidated due to complexity of the reaction system.

The important role of organization of the monomer molecules ${ }^{2,3}$ was substantiated in the thermo-initiated template polymerization as predicted above. The ini- 
tiating radical species is presumed to be produced by reaction between adjacent monomer molecules on the template. $^{19}$

\section{CONCLUSIONS}

The following results were obtained in the present work.

(1) Poly(sodium acrylate) with low $P_{n}$ retards the polymerization of MMAC (the negative template effect), but poly(sodium acrylate) with high $P_{n}$ does not.

(2) The addition of an appropriate amount of sodium chloride to the template polymerization system to increase the ionic strength of the reaction medium enhances the negative template effect even by using a template with high $P_{n}$.

(3) The results obtained are interpretable based on zip and pick-up mechanisms.

(4) MMAC polymerizes thermally in the presence of poly(sodium acrylate) with high $P_{n}$.

\section{REFERENCES AND NOTES}

1. C. M. Paleos ed, "Polymerization in Organized Media," Gordon \& Breach, Philadelphia, PA, 1992.

2. Y. Y. Tan and G. Challa, "Encyclopedia of Polymer Science and Engineering," Vol. 16, Wiley, New York, N.Y., 1989, p 554; Makromol. Chem. Macromol. Symp., 10/11, 215 (1987).

3. Y. Y. Tan, Prog. Polym. Sci., 19, 561 (1994).

4. J. C. Salamone, B. Snider, and W. I. Fitch, J. Polym. Sci., Part $A-1,9,1493$ (1971).

5. V. A. Kabanov, O. V. Kargina, and M. V. Ulyanova, J. Polym. Sci., Polym. Chem. Ed., 14, 2351 (1976).
6. C. Klein, Makromol. Chem., 161, 85 (1972).

7. A. Chapiro, Eur. Polym. J., 9, 417 (1973).

8. C. M. Paleos and P. Dais, J. Polym. Sci., Polym. Chem. Ed., 16, 1495 (1978).

9. S. Aoki and Y. Morimoto, Colloid Polym. Sci., 273, 733 (1995).

10. Y. Yasuda, K. Rindo, R. Tsushima, and S. Aoki, Makromol. Chem., 194, 1893 (1993).

11. Y. Kurokawa, N. Shirakawa, M. Terada, and N. Yui, J. Appl. Polym. Sci., 25, 1645 (1980).

12. J. Matuszewska-Czerwik and S. Polowinski, Eur. Polym. J., 26, 549 (1990).

13. K. Hayakawa, J. P. Santerre, and J. C. T. Kwak, Macromolecules, 16, 1642 (1983).

14. Both propagation and termination rate constants in the template polymerization were lower by several orders of magnitude than those in the polymerization without template, based on increased steric effect and lowered mobility of growing macro-radicals. J.Matuszewska-Czerwik and S. Polowinski, Eur. Polym. J., 27, 1335 (1991)

15. J. Matuszewska-Czerwik and S. Polowinski, Eur. Polym. J., 28, 1481 (1992).

16. S. Aoki and Y. Yasuda, Kobunshi (High Polymers, Jpn.), 43, 539 (1994).

17. Y. Yasuda, K. Rindo, and S. Aoki, Polym. J., 25, 1203 (1993).

18. The thermal polymerization experiments were performed very cautiously to remove oxygen by repeating the following procedure several times; evacuating the space of the glass tube containing the frozen monomer-template solution by applying high vacuum, filling the space by purified nitrogen, defrosting the frozen solution, applying a ultrasonic vibration, and then freezing the contents at dry-ice temperature. When thermal polymerization was attempted by the ordinary method, the polymerization scarcely proceeded.

19. The possibility of radical formation from a peroxide as an unexpected impurity in the monomer or the template was contradicted by the finding that the addition of sodium sulfite as a reducing agent into the polymerization system actually reduced the polymerization rate. 